\title{
Centrifugal Deployment of Membrane with Spiral Folding: Experiment and Simulation*
}

\author{
Nobukatsu OKUIZUMI $^{* *}$ and Takaya YAMAMOTO ${ }^{* * *}$ \\ ${ }^{* *}$ The Institute of Space and Astronautical Science, Japan Aerospace Exploration Agency, \\ 3-1-1 Yoshinodai, Sagamihara, Kanagawa, 229-8510, Japan \\ E-mail: okuizumi@isas.jaxa.jp \\ ***University of Tokyo, 7-3-1 Hongo, Bunkyo-ku, Tokyo, 113-8656, Japan (Currently, Mitsubishi Heavy \\ Industry, 10 Oue, Nagoya, 455-8515, Japan)
}

\begin{abstract}
Centrifugal deployment dynamics of hexagonal membranes stowed with spiral folding are investigated. The centrifugal deployment has a possibility of unfolding large membranes without using extendable masts. Understanding and predicting the deployment dynamics are important to realize large membrane space structures. In this paper, deployment experiments of hexagonal membranes with coarse and fine spiral folding patterns are conducted in vacuum under gravity. Numerical simulations of the centrifugal deployments are also performed employing spring-mass system which models in-plane stiffness of thin membranes and enables fast numerical analysis. Additional modelings taking accounts of buckling, creases, air drag and damping are applied to the spring-mass system model. The experimental and numerical results are compared to examine the characteristics of the deployment dynamics and the validity of the numerical simulation.
\end{abstract}

Key words: Membrane, Centrifugal deployment, Experiment, Simulation, Springmass system

\section{Introduction}

Membrane space structures, such as solar sails, sun shields, thin film solar arrays, etc, have been attracting a lot of attention because of high packaging efficiency and light weight. Recently, solar sails using extremely thin large membranes have been developed in the US, Europe and Japan. The typical concepts of solar sail membrane structures are square membranes stretched by extendable masts and vanes or disk-like membranes deployed by centrifugal force. In Japan, centrifugally deployed solar sails have been studied because of the possibility of unfurling larger membrane than other concepts and folding methods of membranes, dynamics of centrifugal deployment, deployment mechanisms, and so on have been investigated. ${ }^{(1)-(9)}$ Since ground dynamic experiments of huge membranes are not possible, a sounding rocket experiment ${ }^{(2)}$ and small-scale experiments in a vacuum chamber ${ }^{(3),(4)}$ have been conducted. Numerical methods such as spring-mass system models and finite element methods have also been studied to efficiently analyze the behaviors of thin membranes..$^{(3),(7)-(9)}$ Spring-mass system models ${ }^{(7)-(9)}$ simulate in-plane stiffness of thin membranes and enable us to perform fast dynamic simulations. However, the models originally ignore bending stiffness and creases and the buckling strength and damping of the membrane are vague. It will be preferable to take account of them properly to improve the accuracy.

In this paper, centrifugal deployment dynamics of hexagonal membranes stowed with spiral folding ${ }^{(10),(11)}$ are studied. Ground experiments are conducted in a vacuum chamber using small-scale membranes to observe deployment dynamics under a constant rotation 
speed. Numerical simulation is also performed employing the spring-mass system model. Buckling, air drag and damping are considered and a simple model for crease stiffness is newly introduced to evaluate their effects. The experimental and numerical results are compared to discuss deployment behaviors and the validity of the simulation is examined.

\section{Deployment Experiments}

\subsection{Folding method and membrane models}

Deployment experiments of hexagonal membranes with spiral folding ${ }^{(10),(11)}$ are conducted. The spiral folding is used as a test case for the study of centrifugally deployed membranes. Its folding pattern is designed to fold and wind a flat surface into a cylindrical shape by taking account of membrane thickness and calculating quasi-logarithmic-spiral folding lines to avoid generation of wrinkles in stowed shape. Two hexagonal membranes with coarse and fine folding patterns are used in this study. The folding lines of the coarse model are illustrated in Fig. 1. The height in stowed shape and the gaps between folded membrane layers are design parameters. The height corresponds to the distance between folding lines. The deployed and stowed shapes of the experimental models are shown in Figs. 2 and 3. The membrane material is a polyimide film with thickness of $7.5 \mu \mathrm{m}$. Their geometries and the mechanical properties of the material are summarized in Table 1.

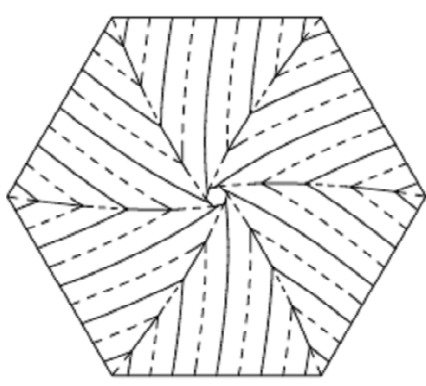

Fig. 1. Folding lines (coarse model). - : mountain folding, - - - : valley folding

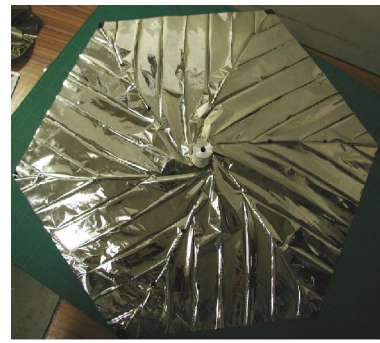

(a) deployed

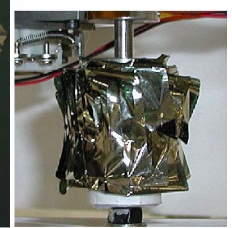

(b) stowed

\begin{tabular}{l|c|c}
\hline Folding pattern & Coarse & Fine \\
\hline Length of diagonal line of hexagon & \multicolumn{2}{|c}{$650 \mathrm{~mm}$} \\
\hline Thickness of membrane & \multicolumn{2}{|c}{$7.5 \mu \mathrm{m}$} \\
\hline Inner radius of stowed shape & \multicolumn{2}{|c}{$20 \mathrm{~mm}$} \\
\hline Height of stowed shape & $45 \mathrm{~mm}$ & $25 \mathrm{~mm}$ \\
\hline Gap between folded membrane layers & $1.0 \mathrm{~mm}$ & $0.3 \mathrm{~mm}$ \\
\hline Young's modulus & \multicolumn{2}{|c}{$3.2 \mathrm{GPa}$} \\
\hline Poisson's ratio & \multicolumn{2}{|c}{0.34} \\
\hline Density & \multicolumn{2}{|c}{$1420 \mathrm{~kg} / \mathrm{m}^{3}$} \\
\hline
\end{tabular}

Fig 2. Coarse folding model.

\subsection{Experimental setup}

The experimental setup is illustrated in Fig. 4. The deployment experiments are performed in a vacuum chamber to reduce the effect of air resistance. The diameter and height of the chamber are $0.66 \mathrm{~m}$ and $1 \mathrm{~m}$, respectively. The air pressure in the chamber is approximately $1 \mathrm{kPa}$. The folded membrane is stowed around a cylindrical hub fixed at the output shaft of a rotation mechanism driven by a stepping motor. The rotation speed can be controlled by a personal computer. In this experiment, the rotation speed is held constant at $3 \mathrm{~Hz}$ in order to reduce deformations due to gravity. The deployment dynamics of the membrane is shot by two cameras placed at the bottom and side of the chamber and 


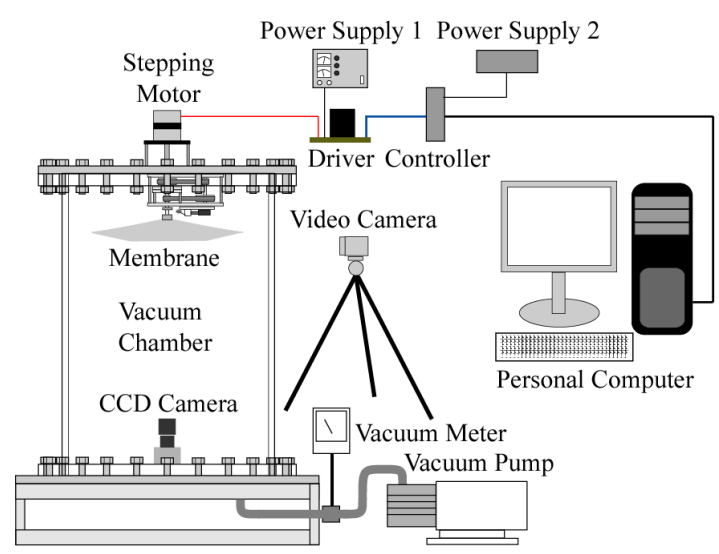

Fig. 4. Experimental setup.

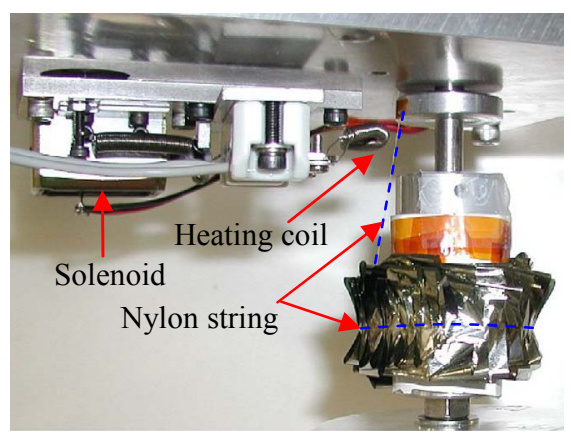

Fig. 5. Release mechanism.

quantified by image processing. Figure 5 shows a release mechanism of the membrane. A nylon string is winded around the membrane, the ends of which are fixed on the parts attached to the rotating shaft. A heating coil is moved by a solenoid and cut the upper end of the string to start deployment.

\subsection{Experiment results}

Figure 6 illustrates bottom and side views of the coarse folding model during deployment, respectively. It is observed that the membranes with spiral folding can be

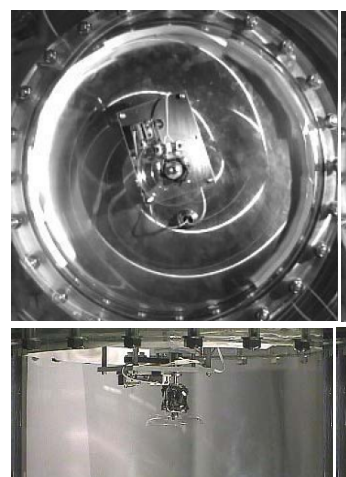

(a) $0.0 \mathrm{sec}$

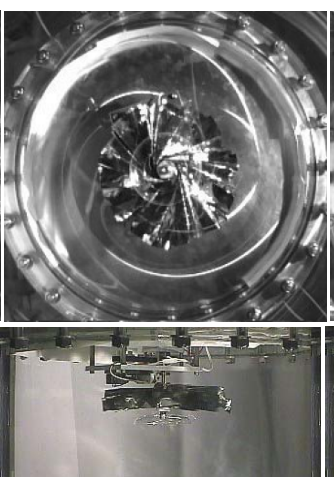

(b) $0.3 \mathrm{sec}$

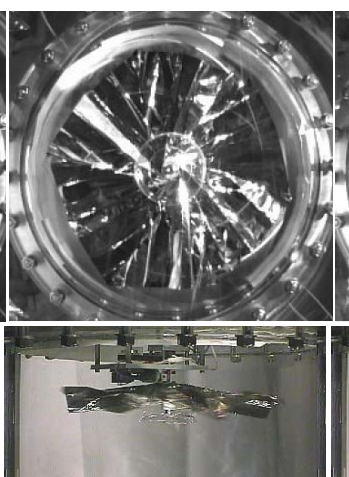

(c) $0.6 \mathrm{sec}$

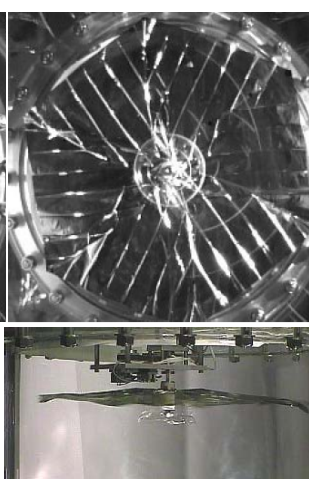

(d) $0.9 \mathrm{sec}$

Fig. 6. Bottom and side views during deployment (coarse folding model).

deployed smoothly. The views from the side camera show that the vertical deflection is small during deployment in spite of gravity. However, both membranes fall down at about 1.3-1.4 seconds for a moment just after maximum deployments as shown in Fig. 7.

Figure 8 shows time histories of deployment rates of the membranes. The deployment rate is the ratio of the maximum radius of the corners of the deploying membrane to the radius of the original hexagon $0.325 \mathrm{~m}$. The membranes deploy almost linearly with time and reach maximum radii in approximately 0.9 seconds. The deployment speeds are almost

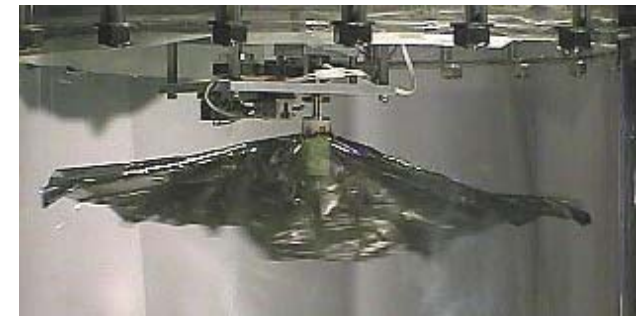

Fig. 7. Falling down at $1.3 \mathrm{sec}$ (coarse model).

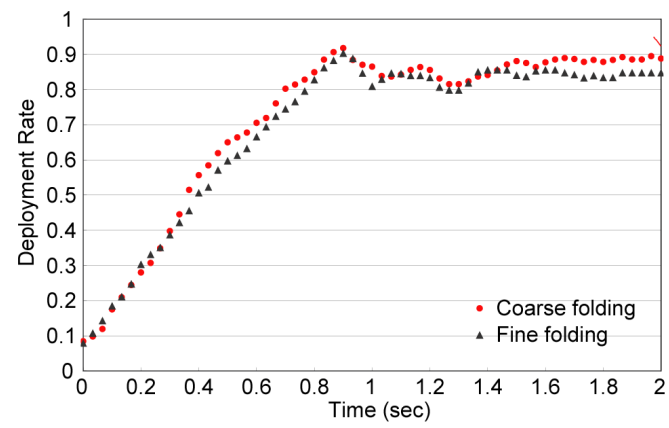

Fig. 8. Time histories of deployment rates. 
independent of the density of folding lines. Deployment rates do not reach 1.0 and the rate of fine folding model is a little smaller. This is probably because the creases inhibit

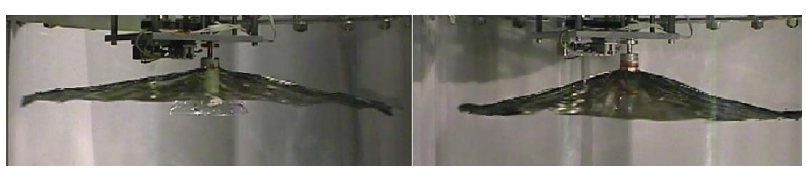

(a) coarse folding model (b) fine folding model

Fig. 9. Side views in steady states

full deployment and the membrane becomes conical due to gravity. It is also noted that the deployment rates fluctuate after 0.9 seconds since the membranes are unrolled excessively after the maximum deployments and in-plane and out-of-plane vibrations are excited. The side views in the steady state after deployment are shown in Fig. 9. The deflection of the fine folding membrane is confirmed to be larger.

\section{Numerical Modeling}

\subsection{Spring-mass system model}

Recently, spring-mass system models have been developed for simple and fast numerical
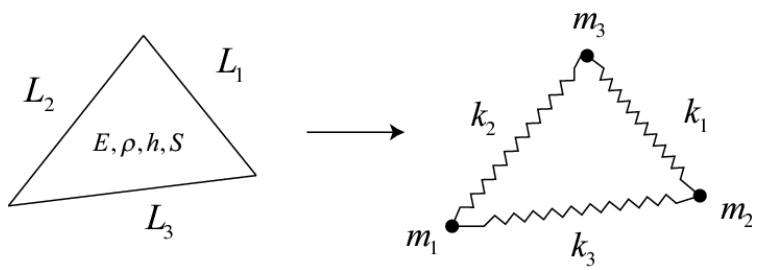
simulations of dynamic behaviors of thin membranes. A spring-mass

Fig. 10. Triangular element.

system model using rectangular element was proposed which approximates stress-strain relationship of elasticity of membrane. ${ }^{(7),(8)}$ Another spring-mass system model using triangular elements based on strain energy consideration was also proposed. ${ }^{(9)}$ Both models consider only in-plane stiffness and ignore bending stiffness. In this paper, the triangular element shown in Fig. 10 is used to discretize hexagonal membranes with curved creases.

In Fig. 10, $E, \rho, h$ and $S$ represent Young's modulus, density, thickness and area of the element, respectively and $L_{i}(i=1,2,3)$ denote natural lengths of the sides of the triangle. The mass of the element $\rho h S$ is equally distributed to the lumped masses $m_{1}, m_{2}$ and $m_{3}$. The spring constants $k_{1}, k_{2}$ and $k_{3}$ are determined so that strain energies of the membrane and the spring-mass system coincide when the element is stretched in the direction parallel to each side and are obtained by Eqs. (1).

$$
\left(\begin{array}{l}
k_{1} \\
k_{2} \\
k_{3}
\end{array}\right)=B_{i j}^{-1}\left(\begin{array}{l}
1 \\
1 \\
1
\end{array}\right), \quad B_{i j}=\frac{p_{i j}^{2} L_{j}^{2}}{E h S}, \quad p_{i j}=1-\frac{4(1+v) S^{2}}{L_{i}^{2} L_{j}^{2}}\left(1-\delta_{i j}\right),
$$

where $v$ and $\delta_{i j}$ denote Poisson's ratio and Kronecker delta, respectively. A spring-mass system model for a membrane is constructed as follows (see Fig.11):

(1) Partition a membrane into triangular elements

(2) Calculate spring constants and masses of each element

(3) Superpose all the springs and masses

(4) Perform additional modeling

(5) Formulate equations of motion

The additional modeling includes buckling strength, crease stiffness, damping and air drag
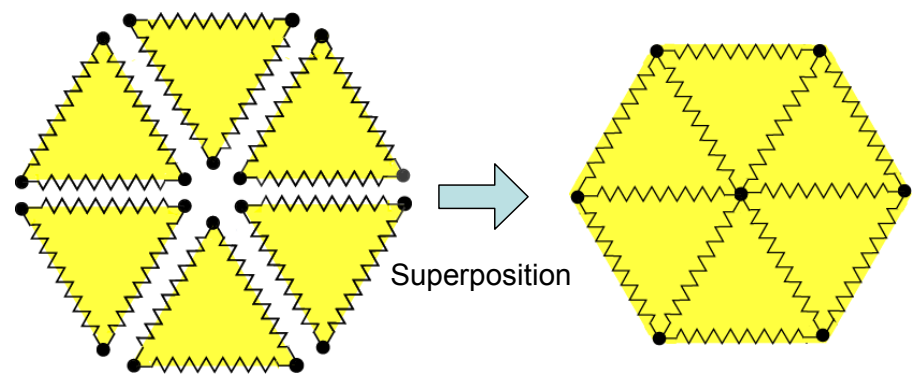

Fig. 11. How to construct a spring-mass system model. 
to roughly evaluate their effects as described below.

\subsection{Buckling strength of springs}

In this study, in order to simulate buckling of thin membranes, the restoring force of a spring is assumed to become constant when the length of the spring is less than a critical value $l_{\mathrm{cr}}$ as shown in Fig. 12. The critical value $l_{\text {cr }}$ is expressed using Euler buckling strength of slender column as:

$$
l_{c r}=L_{i}-\alpha \frac{\pi^{2} h^{2}}{12 L_{i}} .
$$

The coefficient $\alpha$ is determined so that the membrane withstands gravity at the start of deployment as experimental models when centrifugal force is small. If $\alpha$ is not large enough, the membrane collapses as shown in Fig.13.

\subsection{Crease model}

Tensile property of creased thin materials has been studied $^{(12)-(14)}$ to evaluate effective in-plane stiffness. In this research, a simple crease model is newly introduced to the spring-mass system model to take account of the rough effects of creases as follows.

Two triangular elements $\mathrm{ABC}$ and $\mathrm{ABD}$ that contain a crease are considered as shown in Fig.14. It is assumed that the crease generates torque according to the difference between angle $\theta$ and natural crease angle $\theta_{0}$ and that the torque gives forces $F_{C}$ and $F_{D}$ according to the distances between the masses and the crease. $F_{C}$ and $F_{D}$ are assumed to be orthogonal to the triangles and are described by:

$$
F_{C}=\frac{E J l_{A B}\left(\theta-\theta_{0}\right)}{l_{C E}{ }^{2}}, F_{D}=\frac{E J l_{A B}\left(\theta-\theta_{0}\right)}{l_{D F}{ }^{2}},
$$

where $E$ and $J=h^{3} / 12$ denote Young's modulus and geometrical moment of area per unit width, respectively and $l_{\mathrm{AB}}, l_{\mathrm{CE}}$ and $l_{\mathrm{DF}}$ are lengths when the membrane is deformed. Equations (3) were obtained by performing nonlinear finite element analysis of a cantilever beam subjected to a follower force at its end. The summary of the modeling is described in appendix A. Equations (3) give good approximation when the angle is large. $F_{A}$ and $F_{B}$ are forces acting on the masses $\mathrm{A}$ and $\mathrm{B}$ to cancel rigid-body motion of the triangular elements and described in vector form as:

$$
\boldsymbol{F}_{A}=-\frac{l_{B E}}{l_{A B}} \boldsymbol{F}_{C}-\frac{l_{B F}}{l_{A B}} \boldsymbol{F}_{D}, \quad \boldsymbol{F}_{B}=-\frac{l_{A E}}{l_{A B}} \boldsymbol{F}_{C}-\frac{l_{A F}}{l_{A B}} \boldsymbol{F}_{D} .
$$

\subsection{Damping and air drag}

Velocity-proportional dampers are added parallel to the springs to take account of structural damping as shown in Fig.15. The damping coefficients $c_{i}$ are described as:

$$
c_{i}=2 \zeta \sqrt{k_{i} m}, m=\left(m_{j}+m_{k}\right) / 2,(i, j, k=1,2,3),
$$

where $\zeta$ denotes damping ratio. 
In order to take account of residual air in the chamber, the air drag force which act on an element is distributed to three masses. The force applied to a mass is assumed to be

$$
D=\rho_{\text {air }} S_{n} V^{2} / 6,
$$

where $\rho_{\text {air }}, V$ and $S_{n}$ denote the density of air, the average velocity of the masses and the area of element projected to the direction of the velocity, respectively. Although air drag on folded regions may be reduced, it is assumed that the drag acts uniformly for simplicity.

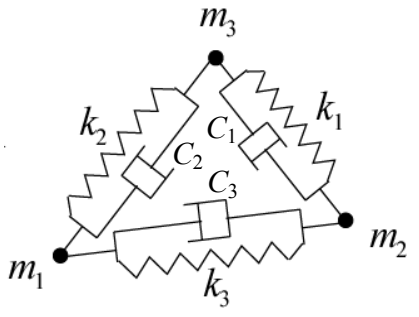

Fig. 15. Spring-mass-damper system.

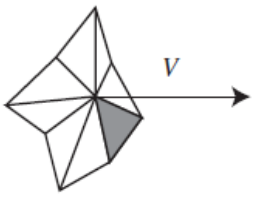

Fig.16. Air drag.

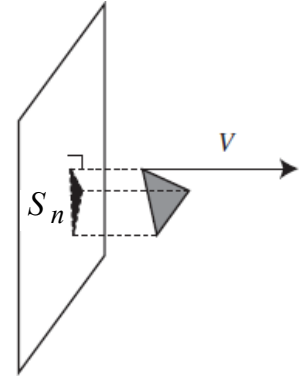

\subsection{Initial radius}

The experimental membrane model in stowed configuration is constricted by the nylon string and the radius is smaller than mathematical geometry of spiral folding. For this reason, initial positions of the masses of numerical model need to be modified as illustrated in Fig. 17. The radii of innermost masses are kept unchanged. The radii of outermost masses are reduced to those of experimental model and the radii of internal masses are reduced proportionally. As a result, the springs are compressed in the initial condition.

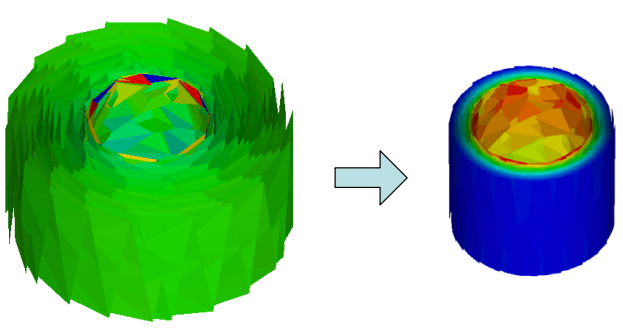

Fig. 17. Adjustment of initial radius.

\subsection{Equation of motion}

The equations of motion of the system are described using rotating cylindrical coordinate system as:

$$
\frac{d^{2}}{d t^{2}}\left(\begin{array}{c}
r \\
\theta \\
z
\end{array}\right)=\left(\begin{array}{c}
\frac{F_{r}}{m}+r \dot{\theta}^{2} \\
\frac{F_{\theta}}{m}-2 \dot{r} \dot{\theta} \\
\frac{F_{z}}{m}
\end{array}\right),
$$

where $F_{r}, F_{\theta}$ and $F_{z}$ denote forces applied to the mass which consists of forces by the springs, creases, damping and air drag. These equations are numerically integrated by the Runge-Kutta method.

\subsection{Numerical models for the experimental membranes}

Figures 18 and 19 show deployed and folded shapes of the hexagonal models discretized with the triangular elements, respectively. Main parameters of the models are shown in Table 2. Natural crease angle is assumed to be

Table 2. Main parameters of simulation models.

\begin{tabular}{c|c|c}
\hline Model & Coarse & Fine \\
\hline Numbers of masses & 2712 & 3816 \\
\hline Number of Elements & 5232 & 7392 \\
\hline Number of springs (edges) & 7944 & 11208 \\
\hline Number of crease edges & 804 & 1416 \\
\hline Natural crease angle & \multicolumn{2}{|c}{$\pi / 4(\mathrm{rad})$} \\
\hline Buckling parameter $\alpha$ & \multicolumn{2}{|c}{100} \\
\hline Damping ratio & \multicolumn{2}{|c}{0.02} \\
\hline
\end{tabular}




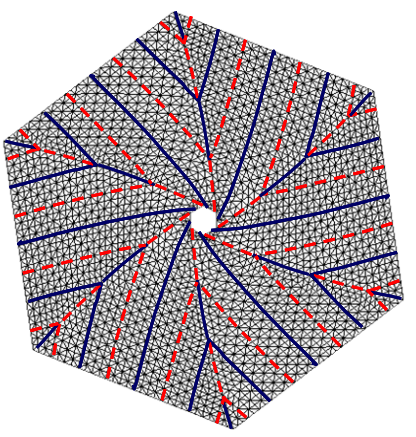

(a)deployed

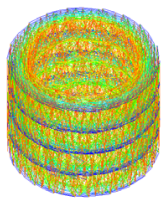

(b) folded

Fig. 18. Spring-mass system model (coarse model).

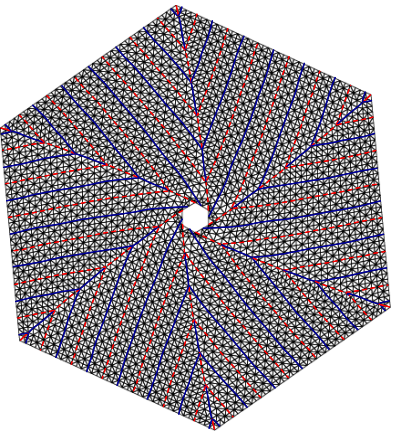

(a)deployed

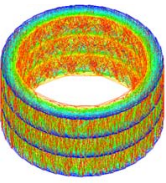

(b) folded

Fig. 19. Spring-mass system model (fine model).

$\pi / 4$. Buckling parameter $\alpha$ was found to be around 100 for both simulation models, with which the models withstand gravity at the start of deployment as experimental models.

\section{Numerical results}

The numerical simulations of the centrifugal deployments were performed employing the spring-mass system models. Figure 20 shows the time histories of deployment rates of basic numerical models in which buckling parameter $\alpha$ equals one and crease stiffness, damping and air drag are ignored. The results of the simulations are rather different from the experiments. Deployment rates vibrate during the deployment and decrease severely after the maximum deployment.

Figure 21 shows the results of additional models compared with experimental results. Buckling strength, crease stiffness, damping and air drag are properly taken into consideration. It is found that the numerical results are significantly improved and in good agreement with the experimental results although numerical models are still deployed a little larger. The deployments progress a little faster than the experiments and maximum deployments are achieved at about 0.82 seconds in both numerical models. The reason for this is probably that the release mechanism takes a little time to release the membranes in

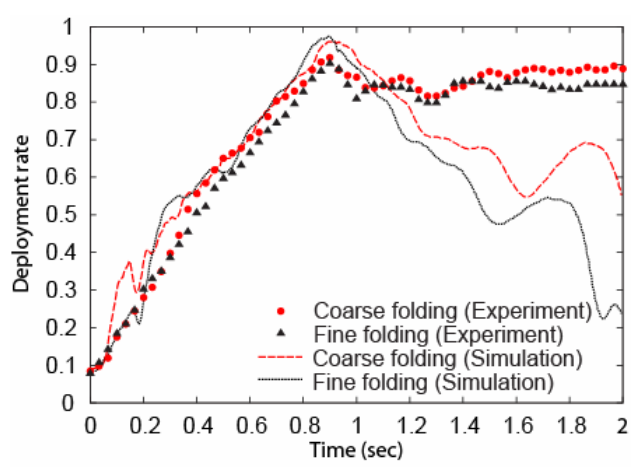

Fig. 20. Comparison of the results of experiments and numerical simulation of basic models.

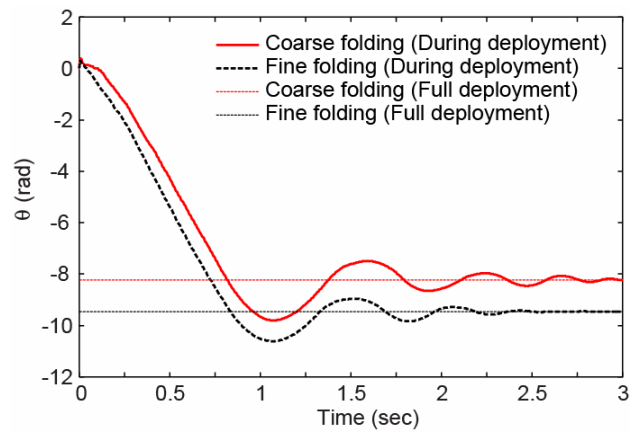

Fig. 22. Variation of average angle of corners.

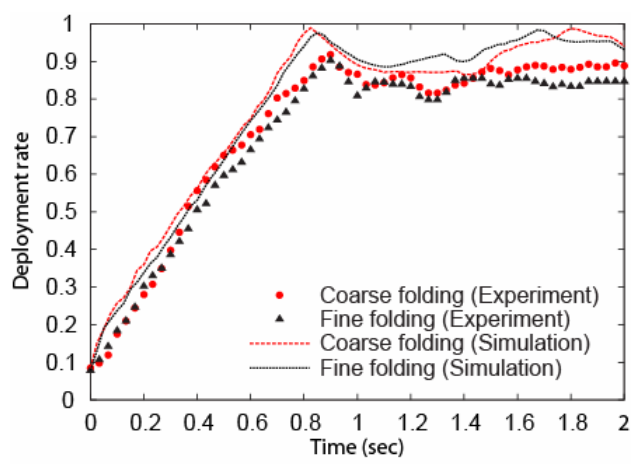

Fig. 21. Comparison of the results of experiments and numerical simulation of detailed models.

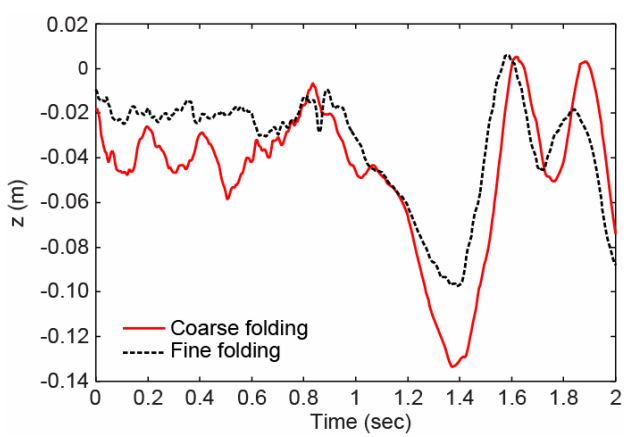

Fig. 23. Average vertical deflection of corners. 
the experiments. The time history of average angle of corners of the hexagon is illustrated in Fig. 22. Thick red and black lines represent the results of the coarse and fine folding models, respectively. Initial angles are aligned to zero. Thin red and black lines represent the angles when full deployment, respectively. The corners are unrolled excessively and overshoot. Then damped in-plane vibrations continue around the full deployment angle. Figure 23 shows average vertical deflections of corners. The corners vibrate with small amplitudes during deployment and fall down after the maximum deployment at 1.3-1.4 seconds as observed in the experiments. Then damped out-of-plane vibrations also continue.

The effects of additional modelings in coarse and fine folding models are displayed in Figs 24 and 25, respectively. The effect of damping ratio is not examined because it is considered sufficiently small and ineffective. Similar tendencies are found in both models. Air drag slows down deployment speeds and moderates vibrations after deployment as we can understand intuitively. The crease stiffness model slightly decreases deployment rates but the differences are not large enough to agree with the experimental results. The buckling strength significantly affects fluctuations during deployment and deployment speeds. Membrane shapes of the coarse folding model are illustrated in Fig. 26. Crease lines are hardly observed in Fig. 26(b) at the maximum deployment because the crease model ignores unrecoverable plastic deformations on crease lines. However, folding lines successfully appears in Fig. 26(c) when the membrane falls down at 1.4 seconds.

The maximum deployments are achieved along with out-of-plain vibrations around the equilibrium shape under gravity and the vibration amplitudes of the numerical models are large enough to reach almost full deployments. Improvement of the crease model and the modeling of bending stiffness may be necessary to suppress excessive deployment rates and
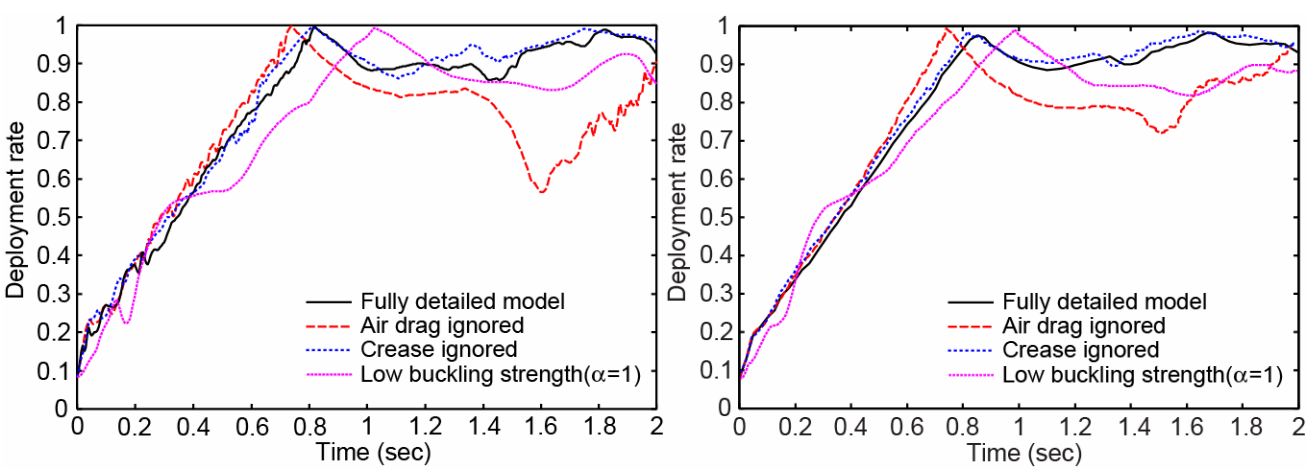

Fig 24. Effect of additional modeling (coarse model). Fig 25. Effect of additional modeling (fine model).

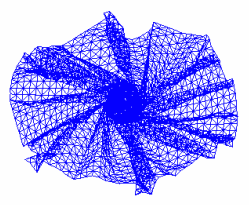

(a) $0.4 \mathrm{sec}$

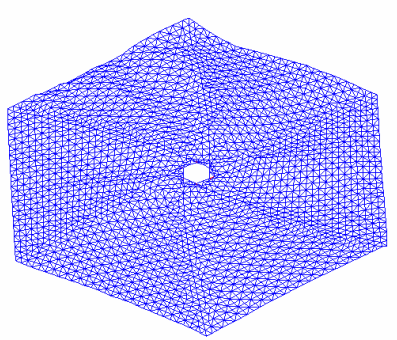

(b) $0.82 \mathrm{sec}$

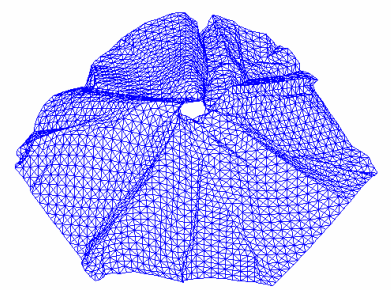

(c) $1.4 \mathrm{sec}$

Fig. 26. Membrane shapes by numerical simulation of coarse folding model.

out-of-plane vibrations. Further study will be necessary on both the experiments and simulations to further improve the difference between the experiments and simulations.

\section{Conclusion}

Centrifugal deployment experiments of the membranes stowed with spiral folding were conducted and compared with the numerical simulations of spring-mass system models. The 
experiments demonstrated that the membranes could be smoothly deployed and that in-plain and out-of-plain vibrations were excited after deployments. It was also confirmed that the spring-mass system models could simulate the deployment dynamics with fairly good accuracy by taking account of the buckling strength, crease stiffness, damping and air drag and by adjusting initial conditions to the experiments.

Vibrations of membranes inevitably occur along with dynamic deployments and the rotating membranes may be unstable in steady state. The understanding of the deployment behaviors and the method to control the dynamics will be important to realize stable membrane structures. For that purpose, the development of fast and reliable numerical simulation methods and a methodology for predicting on-orbit behaviors of the structures based on the numerical simulations and small-scale ground experiments are needed.

Further improvements of the spring-mass system model, experiments and numerical simulations of the deployment dynamics with other folding methods and examination of detailed vibration characteristics of rotating membranes will be the subjects in future.

\section{References}

(1) Kawaguchi, J., "A Solar Power Sail Mission for A Jovian Orbiter and Trojan Asteroid Flybys”, 55th International Astronautical Congress, Vancouver, Canada, pp.1-8, 2004.

(2) Tsuda, Y, et al., "Flight Result and Analysis of Solar Sail Deployment Experiment Using S-310 Sounding Rocket", 56th International Astronautical Congress, Fukuoka, Japan, 2005.

(3) Okuizumi, N., "Deformations and Vibrations of a Rotating Circular Membrane under Distributed Loads", 48th AIAA/ASME/ ASCE/AHS/ASC Structures, Structural Dynamics, and Materials Conference, Honolulu, HI, AIAA-2007-1803, 2007.

(4) Okuizumi, N., Sakamoto, H., Kitajima, A. and Sugita, M., "Centrifugal Deployment Experiments of a Square-shaped Solar Sail Membrane", Proceedings of 18th Workshop on JAXA Astrodymamics and Flight Mechanics, A-5, Sagamihara, Japan, 2008.

(5) Furuya, H. and Inoue, Y., "Dynamic Properties of Rotationally Skew Fold Membrane for Spinning Solar Sail", 47th AIAA/ ASME/ASCE/AHS/ASC Structures, Structural Dynamics, and Materials Conference, Newport, RI, AIAA-2006-1980, 2006.

(6) Kashiwa, M and Onoda, J., "Investigation On Effects of Circumferential Constraint of Membrane on Dynamics of Spinning Solar Sail", 56th International Astronautical Congress, Fukuoka Japan, 2005.

(7) Matsui, T., "Dynamic Analysis of Large Membrane Space Structures Using Particle-System Approximation", Master thesis of the graduate school of the University of Tokyo, 2002 (in Japanese).

(8) Natori, M.C., Nakamura, F. and Okuizumi, N., "Particle System Approximation for Dynamic Behavior of Membranes", International Symposium on Space Technology and Science, 2006-c-11, Kanazawa, Japan, 2006.

(9) Miyazaki, Y. and Iwai, Y., "Dynamics of Membrane Deployed by Centrifugal Force", Space Engineering Conference, Tokyo, Japan, 2005, pp.59-64 (in Japanese).

(10) Watanabe, H., Natori, M., Okuizumi, N., Higuchi, K., "Folding of a Circular Membrane Considering Thickness", Proceedings of 14th Workshop on Astrodymamics and Flight Mechanics, A-4, Sagamihara, Japan, 2004, pp. 19-24.

(11) Natori1, M.C., Kishimoto, N., Watanabe, H. and Higuchi, K., "Morphological Concepts on Efficient Space Structures with Deployable and/or Adaptive Functions", 49th AIAA/ASME/ASCE/AHS/ASC Structures, Structural Dynamics, and Materials Conference, April 2008, Shaumburg, IL, AIAA 2008-2211.

(12) MacNeal, R. and Robbins, W., "Tensile Properties of a Tape with a Transverse Crease", Astro Research Corporation, Rept., ARC-R-241, 1966.

(13) Gough, A., Hossain, A. and Jenkins, C.H., "Experimental and Numerical Study of Creased 
Membranes", 46th AIAA/ASME/ASCE/AHS/ASC Structures, Structural Dynamics, and Materials Conference, April 2005, Austin, TX, AIAA 2005-1976.

(14) Papa, A. and Pellegrino, S., "Systematically Creased Thin-Film Membrane Structures", Journal of Spacecraft and Rockets, Vol.45, No.1, 2008, pp.10-26.

\section{Appendix A. Approximation of crease stiffness model}

In order to obtain an simple creating crease stiffness model, nonlinear finite element analysis of the large deformation of a cantilever beam subjected to follower force at its end was performed as shown in Fig.A1. It was found that the angle $\phi$ of the end was almost proportional to the force $F$ and that the relationship could be approximated by

$$
F \approx \frac{2 E I \phi}{l^{2}}, I=\frac{b h^{3}}{12},
$$

where $E, l, b$ and $h$ are Young's modulus, length, width and height of the beam, respectively. Equation (3) is obtained by letting $\phi=\left(\theta-\theta_{0}\right) / 2, l=l_{C E}$ or $l_{D F}$ and $b=l_{A B}$. In this approximation, the curvature is ignored and the force is evaluated by assuming that the angle at the cusp of crease remains $\theta_{0}$.

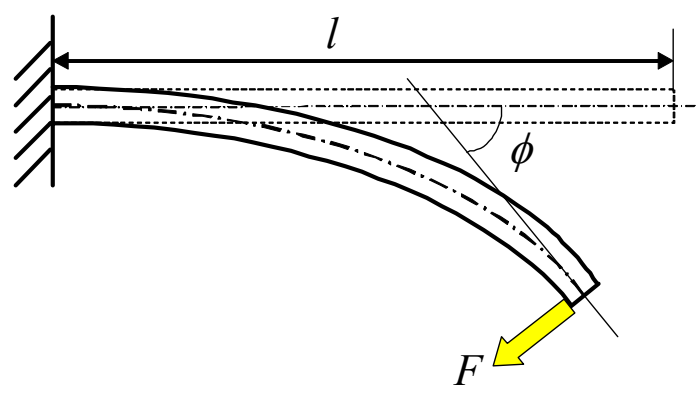

Fig. A1. Large deformation of a cantilever beam. 